Manuscript of the article: Andras Polyak, Lívia Naszályi Nagy, Eszter Drotár, Gabriella Dabasi, Róbert P. Jóba, Zita Pöstényi, Renata Mikolajczak, Attila Bóta, Lajos Balogh: Lu-177-labeled zirconia particles for radiation synovectomy

Appeared in: Cancer Biotherapy and Radiopharmaceuticals (2015) 30(10), 433438, ISSN: 1084-9785 • Online ISSN: 1557-8852, doi: 10.1089/cbr.2015.1881

\title{
Lu-177-labeled zirconia particles for radiation synovectomy
}

Andras Polyak ${ }^{1}$, Lívia Naszályi Nagy ${ }^{2}$, Eszter Drotár ${ }^{2}$, Gabriella Dabasi ${ }^{3}$, Róbert P. Jóba ${ }^{3}$, Zita Pöstényi ${ }^{1}$, Renata Mikolajczak ${ }^{4}$, Attila Bóta ${ }^{2}$, Lajos Balogh ${ }^{1}$

1 National Research Institute for Radiobiology and Radiohygiene (NRIRR), Budapest, Hungary

2 Institute of Materials and Environmental Chemistry, Research Centre for Natural Sciences, Hungarian Academy of Sciences (IMEC RCNS HAS), Budapest, Hungary

3 Department of Nuclear Medicine, Semmelweis University, Budapest, Hungary

4 Radioisotope Centre Polatom, National Centre of Nuclear Research, Otwock, Poland

\begin{abstract}
The present article describes the preparation of beta emitter lutetium-177-lebeled zirconia colloid and its preliminary physico-chemical and biological evaluation of suitability for local radionuclide therapy.

The new ${ }^{177}$ Lu-labeled therapeutic radiopharmaceutical candidate was based on the synthesis mode of a previously described zirconia nanoparticle system. Morphology and size of developed radiopharmaceutical compound were evaluated through scanning electron microscope and dynamic light scattering methods. The radiocolloid had a 1.7 micrometer mean diameter and showed suitable radiolabeling and colloid size in vitro stability at RT and during blood sera stability test. After the in vitro characterizations, the product was investigated in the course of the treatment of a spontaneously diseased dog veterinary patient's hock joint completed with SPECT imaging follow-up measurements and a dualisotope SPECT imaging tests with conventional ${ }^{99 \mathrm{~m}} \mathrm{Tc}-\mathrm{MDP}$ bone scintigraphy. In the treated dog any clinical side effects or signs of histopatological alterations of the joints could not be observed during the treatment. SPECT follow-up studies unequivocally showed appropriately high localization of the ${ }^{177}$ Lu-labeled colloid in hock joint and detectable but negligible radiocolloid leakages in the nearest lymph node, respectively.
\end{abstract}


On the basis of biological follow-up tests orthopaedic team assumed that the ${ }^{177}$ Lu-labeled zirconia colloid based local radionuclide therapy resulted in a significant and long-term improvement in clinical signs of the patient without any remarkable side effects.

\section{Keywords:}

radiosynovectomy, local radiotherapy, radionuclide therapy, ${ }^{177} \mathrm{Lu}$, zirconia , rheumatoid arthritis

Corresponding author: Andras Polyak e-mail: bandi.polyak@gmail.com tel.: +36706225007 fax: +3614822003

postal address: Anna Str. 5, H-1221 Budapest, Hungary 


\section{Introduction}

Localized irradiation of the synovial lining of a joint has emerged as one of the most successful modality for the treatment of rheumatoid arthritis [1]. Local radiation therapy can be effected by intraarticular administration of suitable beta-emitting radionuclides which are bound to colloidal size range particles. This treatment is known as radiosynovectomy (RS) or radiosynoviorthesis (RSV). Numerous beta-emitting (usually lanthanide) radioisotope was used for RSV purpose such as ${ }^{90} \mathrm{Y},{ }^{153} \mathrm{Sm},{ }^{188} \mathrm{Re},{ }^{165} \mathrm{Dy},{ }^{166} \mathrm{Ho},{ }^{169} \mathrm{Er}$ and ${ }^{170} \mathrm{Tm}[2-6]$. We report here the first trials of ${ }^{177} \mathrm{Lu}$ isotope $\left[T_{1 / 2}=6.73 \mathrm{~d}, E_{\beta(\max )}=0.49 \mathrm{MeV}, E_{\psi}=208 \mathrm{keV}(11 \%)\right]$ labeled zirconia based colloid particles: their preliminary physico-chemical and biological evaluation for suitability as a new potential therapeutic agent candidate for radiation synovectomy.

Zirconia, a ceramic material is used for the synthesis of nanoparticle systems $[7,8]$ and for other biomedical applications e.g. in dental implants [9]. It is known to be biocompatible [10, 11], and our preliminary experiments have shown that zirconia is also biodegradable under certain circumstances [12]. Zirconia crystal lattice is known to host yttrium or other lanthanide ions via replacement of $\mathrm{Zr}^{4+}$ ions to $\mathrm{Y}^{3+}$ or $\mathrm{Ln}^{3+}$ ions. This phenomenon is used widely to stabilize monoclinic crystal structure of zirconia during sintering procedures and therefore this characteristics makes zirconia based colloid system suitable for viable binding and carrying of the radiolanthanide ${ }^{177} \mathrm{Lu}$ for the intraarticular injection of therapeutic dose to the joints, as well.

Besides biocompatibility and biodegradability, the most important attributes of radionuclide carrier colloids, microparticulates focusing as the carrier of radionuclides for intra-arterial therapy are appropriate particle size distribution and high radiochemical stability to overcome the possible in vivo leaching of the radioisotope [13]. In the present study, we have explored the possibility of a zirconia particulate system above one micrometer effective mean particle size.

In this present study the new product was investigated in the course of the radionuclide therapy of a spontaneously diseased dog veterinary patient's hock joint. As previously, the objective of treatment of a veterinary patient was to provide a preliminary preclinical proof of concept of a potential prospective human medical application and pharmaceutical before the further detailed evaluations $[12,14,15]$. 


\section{Materials and methods}

\section{Chemicals}

${ }^{177} \mathrm{Lu}$ isotope (radiochemical purity $>99.9 \%$ ) was obtained from the Radioisotope Centre Polatom, Poland in the form of $0.04 \mathrm{M} \mathrm{HCl}$ solution of lutetium chloride $\left(\mathrm{LuCl}_{3}\right)$. For the control examination, lyophilized MDP kit was obtained from Medi-Radiophamra Co. Ltd., Hungary. Absolute ethanol (a.r., $>99.7 \%,<0.2 \%$ water, Reanal), cesium chloride $(\mathrm{CsCl}$, Specpure, trace metal basis); zirconium(IV) butoxide (TBOZ, 80 wt\% in butanol, Sigma-Aldrich) were used for $\mathrm{ZrO}_{2}$ synthesis. TBOZ was kept and manipulated under argon gas. Ultrapure water was collected from a MilliQ System. HEPES buffer solution (1M, 59205C) was purchased from Sigma-Aldrich.

\section{Preparation of ${ }^{177}$ Lu-labeled zirconia particles}

The ${ }^{177} \mathrm{Lu}$-labeled colloid was based on previously prepared and described zirconia nanoparticle system $[16,17]$. Briefly, $100 \mathrm{ml}$ of abs. ethanol was stirred at $300 \mathrm{rpm}$ and heated to $60^{\circ} \mathrm{C}$ in a closed glove box flushed with argon gas. $0.4 \mathrm{ml}$ of freshly prepared $0.1 \mathrm{M} \mathrm{CsCl}$ solution was pipetted into the ethanol. After homogenization $3.25 \mathrm{ml}$ of TBOZ was quickly added to it and the glass vial was closed. The solution became white within one minute. The sol was centrifuged three times at $4000 \mathrm{rpm}$ (10 $\mathrm{min})$. The supernatant was separated and the sediment was resuspended in ethanol by an ultrasonic homogenizer (150VT, BioLogics). The obtained $\mathrm{ZrO}_{2}$ sol was dried at $60^{\circ} \mathrm{C}$ and stored in powder form. Before the radiolabeling, 0.1 mg of it was resuspended in $300 \mu l 0.01$ M HEPES buffer $(\mathrm{pH} 7.0)$ and ultrasonicated for 30 $\min$. Then $5 \mu \mathrm{l}$ of ${ }^{177} \mathrm{LuCl}_{3}$ in $0.04 \mathrm{M} \mathrm{HCl}$ (activity: $316 \mathrm{MBq}$ ) and $50 \mu \mathrm{l} 0.1 \mathrm{M}$ HEPES buffer was added to the resuspended colloid and the ${ }^{177}$ Lu containing colloid suspension was incubated at RT for 15 minutes.

\section{In vitro characterization of labeled zirconia particles}

To evaluate its in vitro stability, ${ }^{177}$ Lu-labeled zirconia colloid was kept at $21^{\circ} \mathrm{C}$ up to 1 week after the preparation and particle size distribution and ${ }^{177}$ Lu-labeling yield were investigated after 1, 8, 24 and 168 hours. Labelling efficiency was examined by thin layer chromatography (ITLC-SG, silica gel impregnated glass fiber plates, Gelman Sciences Inc). In vitro serum stability was evaluated in canine blood serum. A $20-\mu \mathrm{L}$ aliquot of radiolabeled product was added to $500 \mu \mathrm{L}$ of serum. Sample was stored at $37^{\circ} \mathrm{C}$ and radioabeling yield was evaluated at $1,8,24$ and 168 hours of incubation using the same ITLC-SG method. 
Morphological investigation of the zirconia particles was carried out on a ZEISS EVO 40XVP scanning electron microscope (SEM, tungsten cathode, $20 \mathrm{kV}$ accelerating voltage). Diluted sample of the original zirconia sol was dropped and dried on carbon coated copper grids. Particle size distribution measurements were carried out in an Avid Nano w310i dynamic light scattering (DLS) instrument equipped with a fiber coupled laser diode $(\lambda=660 \mathrm{~nm})$ and a silicon avalanche photodiode detector at fixed angle of $90^{\circ}$. Input parameters were: solvent refractive index: 1.330 , viscosity: 0.891 Pas, temperature: $25^{\circ} \mathrm{C}$, laser power: $25 \%$, optical attenuation: 3\%. 10 runs of 10 seconds were recorded. The number weighted size distribution function is presented. The suspension was ultrasonicated for $5 \mathrm{~min}$ before the measurement.

\section{Biological evaluation of prepared compound}

An eleven-year old spayed Cavalier King Charles Spaniel was referred into our Institute with chronic, destructive, therapy-resistant osteoarthritis in the left hock joint. The dog had been walking 3-legged for 6 months, and the handling ortopaedic veterinary-team offered amputation to the owners. The patient's blood tests showed increased white cell number (19.6 G/I) with lymphocytosis $(5.0 \mathrm{G} / \mathrm{l}$ ) and elevated CRP (C-reactive protein: $28 \mathrm{mg} / \mathrm{l}$ ) and ALP (alkaline phosphatase: $613 \mathrm{lU} / \mathrm{I}$ ) levels. These latter findings and the physical examinations revealed painful, swelling hock joint and atrophic muscles in the left hind-leg.

The radionuclide therapy was performed by injecting $200 \mu \mathrm{l}$ of radiolabeled compound intraarticularly directly to the left hock joint of the dog. The $178 \mathrm{MBq}$ injected activity was selected by the extrapolation from the human related EANM procedure guidelines [18]. After intraarticular application of compound the hock joint was immobilized with a bandage, and the animal was taken into a cage for 3 days and only short walks were allowed for her. Bandage and the overall physical conditions (and joint flexibility) were checked twice daily.

Serial whole body SPECT ("single-photon emission computed tomography") images were made by AnyScan SPECT camera (Mediso Inc. HU) 1, 8, 24 hours and 1 week, 1 month and 3 months post administration to detect the potential leakage of activity from the hock joint and so the potential degradation of the colloidal product before the proper time. 24 hours, 1 week and 1 month post-injection control SPECT examination was performed by dual-isotope SPECT imaging: before the SPECT investigations ${ }^{99 \mathrm{~m}} \mathrm{TC}$-MDP ("Methanediphosphonic acid, Medronic acid") injection (Medi-Radiopharma Co. Ltd., Hungary) was applied intravenously in the patient and the localizations and biodistributions of the two radiopharmaceuticals were recorded simultaneously. Resulted SPECT/CT images were processed by ROI / VOI ("region of interest", "volume of interest") analysis using Interview Software (Mediso Ltd. Hungary). 
Besides the SPECT imaging follow-up, serial blood and urine samples were collected and analyzed to detect potential excretion ratio of injected radioactivity through these pathways. 1, 3 days, 1,2,4 weeks and 3 months post-treatment blood samples were also collected for carrying out haematological, biochemical and Comet assay studies to check the possible radiotoxicological effects. (Haem. parameters studied: WBC, RBC, nemoglobin, hematocrit (PCV), MCV, $\mathrm{MCH}, \mathrm{MCHC}$, platelets, neutrophils, lymphocytes, eosinophils, monocytes, basophils; Biochem. parameters studied: AST, ALT, ALP, total bilirubin, total prot, alb, glob, alfa amilase, glu, chol, urea, creatinine, $\mathrm{P}, \mathrm{Na}, \mathrm{K}, \mathrm{Cl}, \mathrm{Ca}$.)

The treated animal was kept in compliance with all applicable sections of the Hungarian Laws No. XXVIII/1998 and LXVII/2002 on the protection and welfare of animals and animal welfare directions and regulations of the European Union. The study was also approved by the Governmental Ethical Committee (permission No. 22.1/609/001/2010). The informed owners of the only referred dog patient declared their consent to the study.

\section{Results and Discussion}

\section{In vitro characterization results}

Morphology and particle size distribution of the product were examined through SEM and DLS and it could be concluded that zirconia particles, which were originally nearly $380 \mathrm{~nm}$ of diameter [17] were partially dispersed in HEPES buffer, and by this means we could obtain loosely packed inorganic aggregates (Fig 1). Over micron size aggregates remain in the slurry with a mean diameter of 1.7 micrometer according to the DLS size distribution function (Fig 2).The resulted aggregates possessed higher surface area $\left(16 \mathrm{~m}^{2} / \mathrm{g}\right)$ [17] and lower solid content than single zirconia particles of the same diameter (specific surface area $\approx 11 \mathrm{~m}^{2} / \mathrm{g}$ ) [16]. The advantage of such a construction against compact particles of micron-sized diameter is: lower quantity of extraneous material applied regarding to larger-colloid surface area for the grafting of ${ }^{177}$ Lu radionuclides (higher drug/carrier dose). The lower solid content may be of interest for the long-term degradation and elimination of the carrier colloid, as well.

The radiolabeled colloid showed high in vitro radiochemical and colloid size stability. The labeling efficiency of the sample incubated at room temperature was found to be above $99 \%$ after 1,8 and 24 hours and $97 \%$ after 1 week of storage. ${ }^{177}$ Lu-labeled particles had durable blood serum stability during the 1 -week incubation. The ${ }^{177}$ Lu-bearing yield was found to be 
$>99 \%$ after 1,8 , and 24 hours and 98\% 1 week postlabeling. DLS measurements proved that the storage at RT did not cause significant change in particle size distributions. The mean hydrodynamic diameter values of the colloidal product were found in the range of 1.4-2.5 $\mu \mathrm{m}$ during the 1-week follow-up. Results suggested that ${ }^{177}$ Lu-labeled zirconia particles do not disintegrate under the effect of self-beta-radiation or by the storing in different physiological conditions at different temperatures.

\section{Results of biological study}

In the treated dog no clinical side effects were recorded during the treatment. The SPECT images were recorded at 1, 8, 24 hours and 1 week, 1 month and 3 months postadministration time points. SPECT follow-up studies unequivocally proved the localization of the ${ }^{177}$ Lu-labeled particles in the hock joint as well as negligible leakages (under $1 \%$ of total injected activity was measured by ROI/VOI analyses) of the radiocolloid in the nearest lymph node. Figure 3 and Figure 4 represent dorsoventral dual-isotope SPECT images of the veterinary patient recorded simultaneously 24 hours and 1 month post-administration using ${ }^{99 \mathrm{~m}} \mathrm{Tc}-\mathrm{MDP}$ as control radiopharmaceutical. Images "A" of Fig 3 and Fig 4 show the only detectable source of the ${ }^{177} \mathrm{Lu}$ in the whole body SPECT scans, while images "B" show parallel recorded whole body ${ }^{99 \mathrm{~m}} \mathrm{Tc}-\mathrm{MDP}$ distributions. The exact localization the hock joint is signed by retrospectively added reference lines in the paired scans. Investigations of blood and urine samples have confirmed the lack of leakage of the radiocolloid, also only insignificant ratios of activity (under $0.01 \%$ values of total ID) were found in the collected samples. Moreover, no remarkable differences could be perceived in haematological and biochemical parameters as well as in Comet assays before and after the application. All these biological evaluation indicates that the ${ }^{177}$ Lu-labeled zirconia colloid had no adverse effects and thus might be acceptable for further evaluation.

Three days after treatment bandage was removed and the patient was agitated progressively to move. One week to 3 months after completion of treatment there was a significant improvement in clinical symptoms eg.: the dog could walk hours with only a little lame, no swelling, joint flexibility as in contra lateral hock joint. Follow-up bone scintigrams 1 month and 3 months post-treatment revealed normal ${ }^{99 \mathrm{~m}} \mathrm{Tc}-\mathrm{MDP}$ radiopharmaceutical uptakes (see fig 4 image "B" and fig 5). On the basis of follow-up tests and owner's semi-quantitative evaluations orthopaedic team assumed that local ${ }^{177}$ Lu-labeled zirconia colloid based radionuclide therapy resulted a significant and long-term improvement in clinical signs without any remarkable side effects. 


\section{Conclusions}

The aims of our experiments were to produce a novel ${ }^{177}$ Lu-bearing therapeutic radiopharmaceutical candidate using biocompatible, inorganic carrier colloid, which may function as a potential alternative to other radiocolloidal agents applied in radiation synovectomy at present. The ${ }^{177}$ Lu-labeled colloid was prepared following a simple radiolabeling method of a previously originated zirconia nanoparticle system. The new agent was had excellent in vitro ${ }^{177}$ Lu-bearing and colloidal stability. The product was investigated in the course of treatment of a spontaneously diseased dog veterinary patient. In vivo studies also proved good stability of the product by the clearly visible and detectable high ratio of retained activity in the treated hock joint during the one week follow-up. The present preliminary findings showed the potential of the novel product towards its application as an alternative compound for radiation synovectomy.

\section{References}

1. Siegel, M.E., H.J. Siegel, J.V. Luck Jr, Radiosynovectomy's clinical applications and cost effectiveness: A review. Seminars in Nuclear Medicine, 1997. 27(4): p. 364-371.

2. Polyak, A., T. Das, S. Chakraborty, R. Kiraly, G. Dabasi, R.P. Joba, C. Jakab, J. Thuroczy, Z. Postenyi, V. Haasz, Thulium-170-Labeled Microparticles for Local Radiotherapy: Preliminary Studies. Cancer Biotherapy and Radiopharmaceuticals, 2014.

3. Máthé, D., P.R. Chaudhari, L. Balogh, A. Polyák, R. Király, R. Glávits, G. Andócs, E. Perge, G.A. Jánoki, Preliminary studies with ${ }^{188}$ Rhenium-tin colloid for radiation synovectomy: preparation, size determination, in vivo distribution, effects and dosimetry studies. Nucl Med Rev Cent East Eur, 2002. 5: p. 89-104.

4. van der Zant, F.M., R.O. Boer, J.D. Moolenburgh, Z.N. Jahangier, J.W. Bijlsma, J.W. Jacobs, Radiation synovectomy with (90)Yttrium, (186)Rhenium and (169)Erbium: a systematic literature review with meta-analyses. Clin Exp Rheumatol, 2009. 27(1): p. 130-9.

5. Torres, M., E. Ayra, O. Albuerne, M.A. Montano Delgado, Absorbed dose profiles for (32)P, (90)Y, (188)Re, (177)Lu, (153)Sm and (169)Er: radionuclides used in radiosynoviortheses treatment. Rev Esp Med Nucl, 2009. 28(4): p. 188-92.

6. Chinol, M., S. Vallabhajosula, S.J. Goldsmith, M.J. Klein, K.F. Deutsch, L.K. Chinen, J.W. Brodack, E.A. Deutsch, B.A. Watson, A.J. Tofe, Chemistry and biological behavior of samarium-153 and rhenium-186-labeled hydroxyapatite particles: potential radiopharmaceuticals for radiation synovectomy. J Nucl Med, 1993. 34(9): p. 1536-42.

7. Masoodiyeh, F., J. Karimi-Sabet, A.R. Khanchi, M.R. Mozdianfard, Zirconia nanoparticle synthesis in sub and supercritical water - particle morphology and chemical equilibria. Powder Technology, 2015. 269(0): p. 461-469.

8. Manoharan, D., A. Loganathan, V. Kurapati, V.J. Nesamony, Unique sharp photoluminescence of size-controlled sonochemically synthesized zirconia nanoparticles. Ultrasonics Sonochemistry, 2015. 23(0): p. 174-184. 
9. Konstantinidis, I.K., S. Jacoby, M. Rädel, K. Böning, Prospective evaluation of zirconia based tooth-and implant-supported fixed dental prostheses: 3-Year results. Journal of Dentistry, 2015. 43(1): p. 87-93.

10. Kohal, R.J., M. Bachle, W. Att, S. Chaar, B. Altmann, A. Renz, F. Butz, Osteoblast and bone tissue response to surface modified zirconia and titanium implant materials. Dent Mater, 2013. 29(7): p. 763-76.

11. Hisbergues, M., S. Vendeville, P. Vendeville, Zirconia: Established facts and perspectives for a biomaterial in dental implantology. J Biomed Mater Res B Appl Biomater, 2009. 88(2): p. 519-29.

12. Polyák, A., L. Naszalyi Nagy, A. Bota, G. Dabasi, P. Joba, G. Trencsenyi, Z. Postenyi, V. Haasz, G. Jánoki, G. Jánoki, L. Balogh, First biological applications of Tc-99m labelled complex silica@zirconia@poly(L-malic acid) theranostic nanoparticles in Eur J Nucl Med Mol Imaging. 2014;41:442.

13. Nijsen, J.F., A.D. van het Schip, W.E. Hennink, D.W. Rook, P.P. van Rijk, J.M. de Klerk, Advances in nuclear oncology: microspheres for internal radionuclide therapy of liver tumours. Curr Med Chem, 2002. 9(1): p. 73-82.

14. Polyak, A., I. Hajdu, M. Bodnar, G. Dabasi, R.P. Joba, J. Borbely, L. Balogh, Folate receptor targeted self-assembled chitosan-based nanoparticles for SPECT/CT imaging: Demonstrating a preclinical proof of concept. Int J Pharm, 2014. 474(1-2): p. 91-94.

15. Balogh, L., J. Thuroczy, G. Andocs, D. Mathe, P. Chaudhari, E. Perge, I. Biksi, A. Polyak, R. Kiraly, G.A. Janoki, Sentinel lymph node detection in canine oncological patients. Nucl Med Rev Cent East Eur, 2002. 5(2): p. 139-44.

16. Widoniak, J., S. Eiden-Assmann, G. Maret, Synthesis and Characterisation of Monodisperse Zirconia Particles. European Journal of Inorganic Chemistry, 2005. 2005(15): p. 3149-3155.

17. Naszályi-Nagy, L., J. Mihály, A. Polyák, B. Debreczeni, B. Császár, I. Szigyártó, A. Wacha, Z. Czégény, E. Jakab, S. Klébert, E. Drotár, G. Dabasi, L. Balogh, A. Bóta, K. É, Inherently fluorescent and porous zirconia colloid: preparation, characterization and drug adsorption studies. Journal of Materials Chemistry B, 2015;3:7529.

18. Clunie, G., M. Fischer, EANM procedure guidelines for radiosynovectomy. Eur J Nucl Med Mol Imaging, 2003. 30(3): p. Bp12-6. 


\section{Figures}

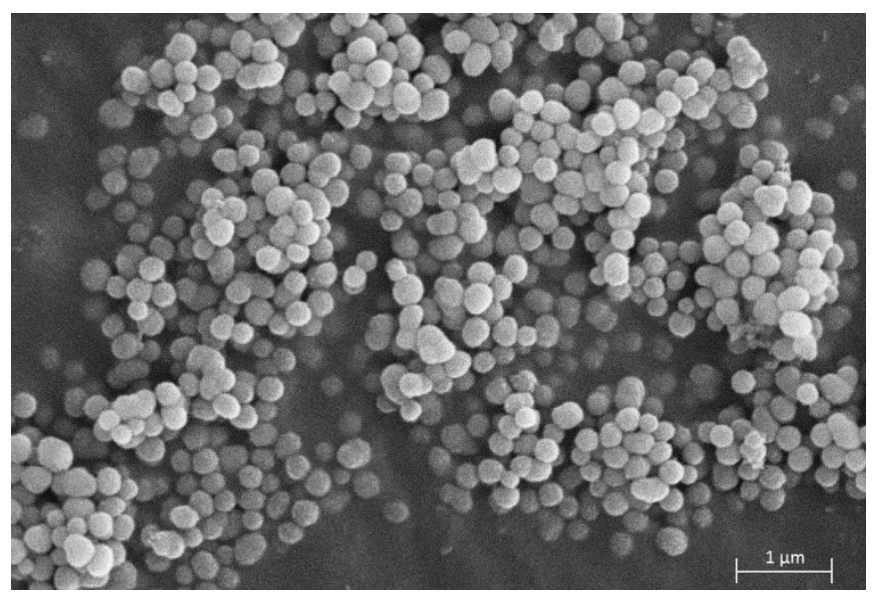

Figure 1. SEM images of ${ }^{177}$ Lu labeled zirconia colloid: partially packed nanoparticular aggregates are observable



Figure 2. The resulted effective particle size distribution of ${ }^{177}$ Lu labeled zirconia colloid: mean diameter $\mathrm{MD}=1.7 \mu \mathrm{m}$ 
${ }^{177}$ Lu-labeled zirconia particles for radiosynovectomy

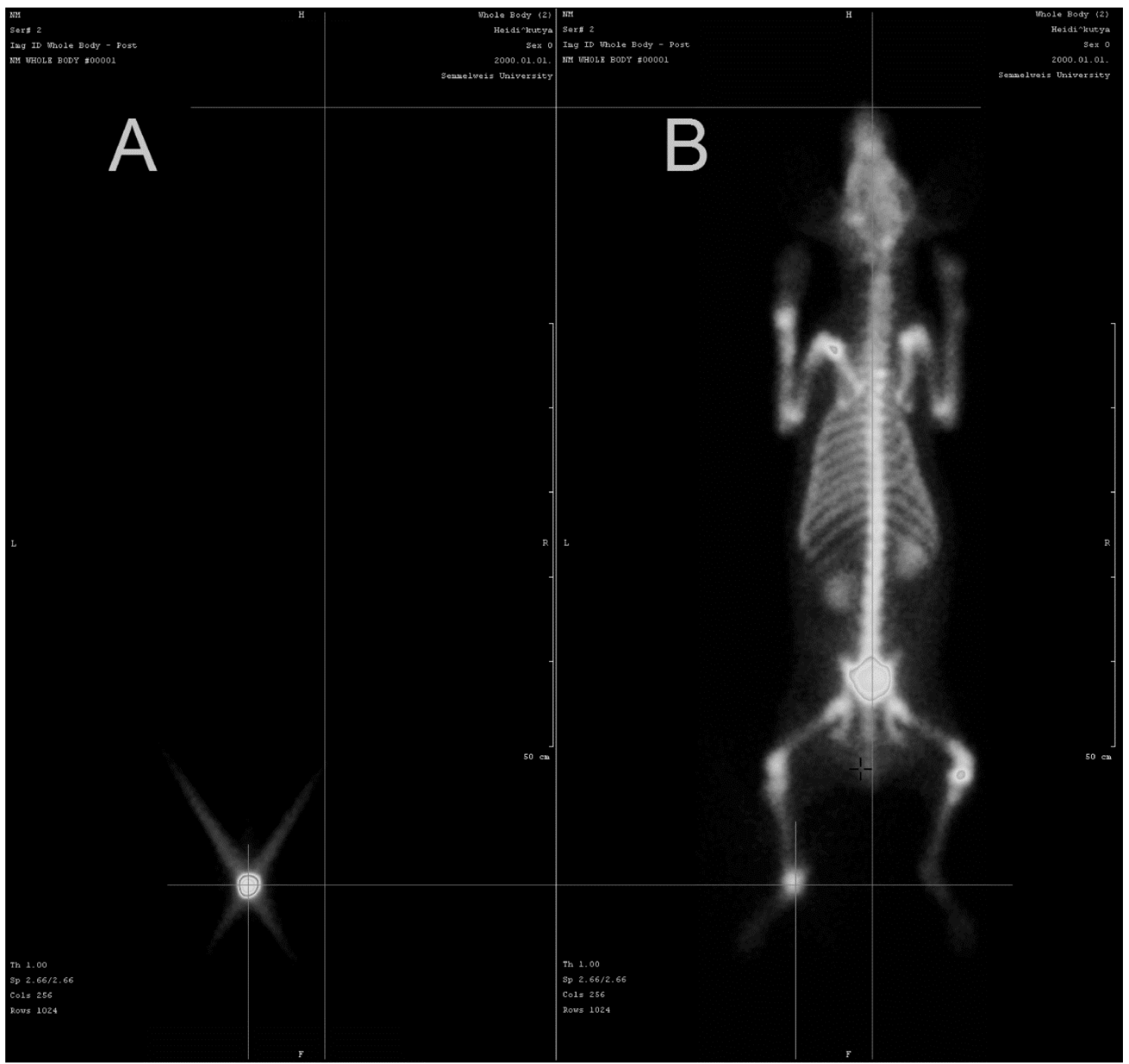

Figure 3. Dual isotope SPECT images of dog veterinary patient. A: ${ }^{177}$ Lu labeled zirconia colloid injected intraarticularly into the left hock joint, 24 hour post injection; B: control ${ }^{99 \mathrm{~m}} \mathrm{Tc}-\mathrm{MDP}$ radiopharmaceutical injected i.v. 1 hour post injection 
${ }^{177}$ Lu-labeled zirconia particles for radiosynovectomy

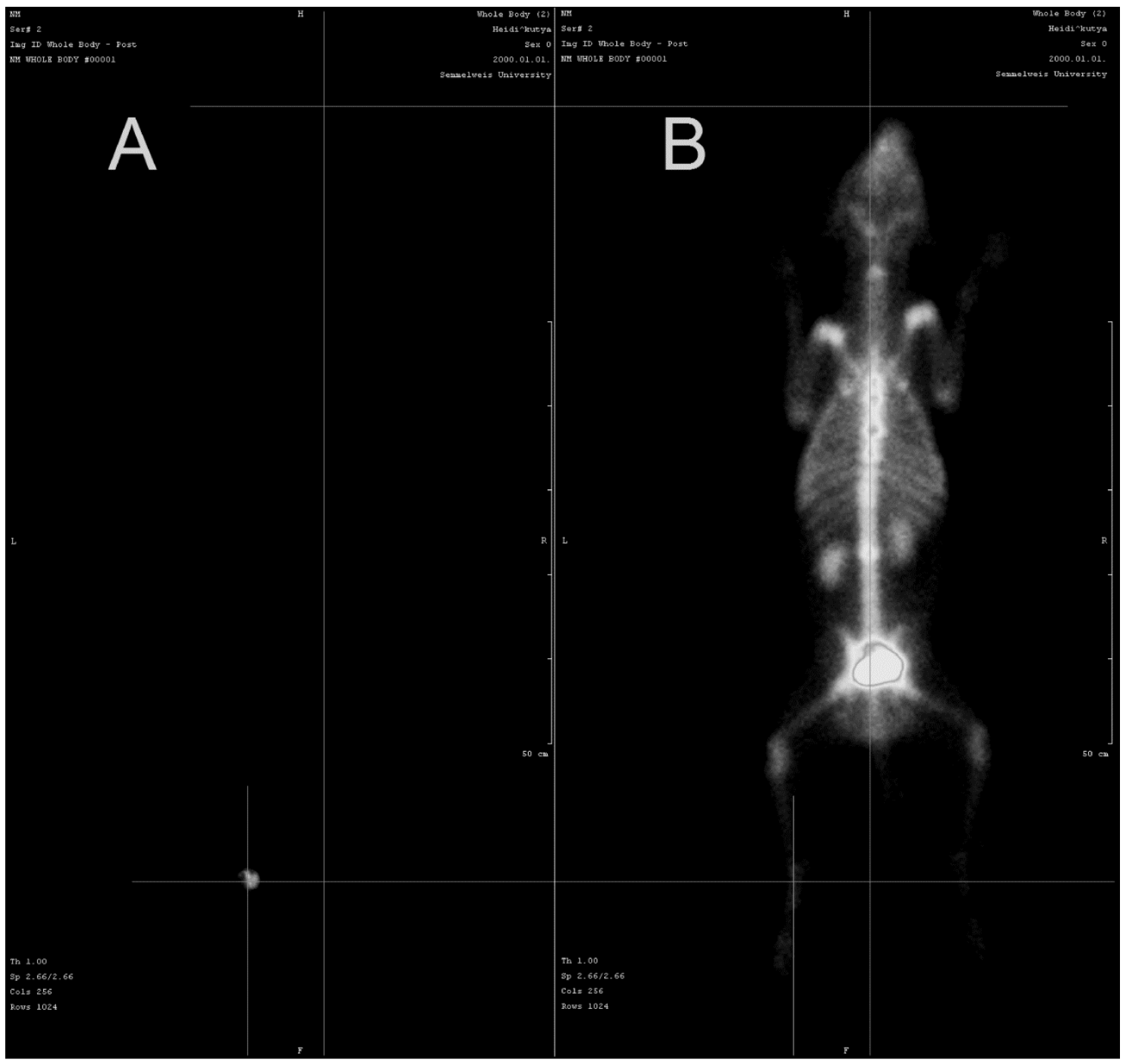

Figure 4. Dual isotope SPECT images of dog veterinary patient. A: ${ }^{177}$ Lu labeled zirconia colloid injected intraarticularly into the left hock joint, 1 month post injection; B: control ${ }^{99 \mathrm{~m}}$ Tc-MDP radiopharmaceutical injected i.v. 1 hour post injection 
${ }^{177}$ Lu-labeled zirconia particles for radiosynovectomy

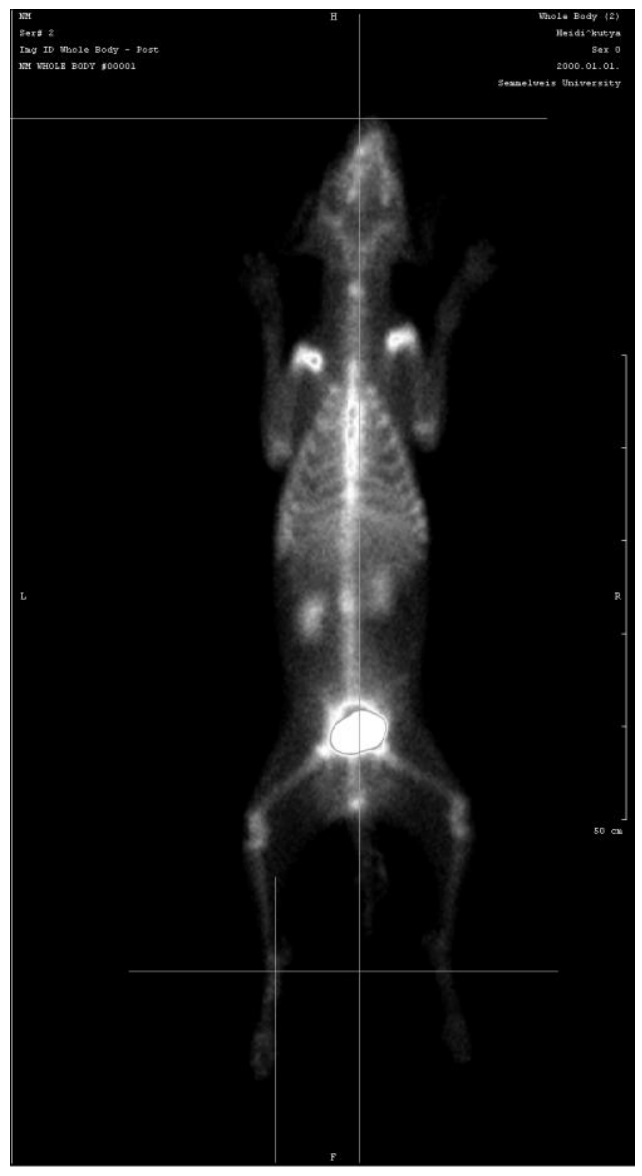

Figure 5. ${ }^{99 \mathrm{~m}} \mathrm{Tc}-\mathrm{MDP}$ whole body SPECT scan of dog veterinary patient 3 months post treatment. 